\title{
Hénon mappings with biholomorphic escaping sets
}

\author{
Sylvain Bonnot ${ }^{1 *} \mathbb{D}$, Remus Radu² and Raluca Tanase ${ }^{2}$
}

\author{
${ }^{*}$ Correspondence: \\ sylvain@ime.usp.br \\ ${ }^{1}$ Mathematics and Statistics \\ Institute, University of Sao \\ Paulo, Rua do Matão 1010, \\ São Paulo 05508-090, Brazil \\ Full list of author information \\ is available at the end of the \\ article
}

\begin{abstract}
For any complex Hénon map $H_{P, a}:\left(\begin{array}{l}x \\ y\end{array}\right) \mapsto\left(\begin{array}{c}P(x)-a y \\ x\end{array}\right)$, the universal cover of the forward escaping set $U^{+}$is biholomorphic to $\mathbb{D} \times \mathbb{C}$, where $\mathbb{D}$ is the unit disk. The vertical foliation by copies of $\mathbb{C}$ descends to the escaping set itself and makes it a rather rigid object. In this note, we give evidence of this rigidity by showing that the analytic structure of the escaping set essentially characterizes the Hénon map, up to some ambiguity which increases with the degree of the polynomial $P$.
\end{abstract}

Keywords: Hénon mappings, Escaping sets, Biholomorphisms

\section{Introduction}

We recall that complex Hénon mappings are polynomial automorphisms of $\mathbb{C}^{2}$ given by the formula:

$$
H_{P, a}:\left(\begin{array}{l}
x \\
y
\end{array}\right) \mapsto\left(\begin{array}{c}
P(x)-a y \\
x
\end{array}\right), \quad \text { where } P \in \mathbb{C}[X] \text { and } a \in \mathbb{C}^{*} .
$$

One can always assume that the polynomial $P(X)$ of degree $d$ is monic and centered (i.e., the coefficient of degree $d-1$ is zero). Any such map has constant Jacobian, equal to $a$.

Those maps were first studied in [5-7]. The behaviour at infinity has been addressed in [8] and the structure of the associated Julia sets has been considered in a long series of articles by Bedford and Smillie, culminating in $[1,2]$. We define the escaping set $U^{+}$to be the set of all points of $\mathbb{C}^{2}$ with unbounded forward orbit.

The whole purpose of this article can be summarized as follows: can one recover a Hénon map from the knowledge of the analytic structure of its escaping set? This question was first formulated by Hubbard, based on the explicit formulas of [6]. Our study is built on the methods first developed by Bousch in his unpublished manuscript [3].

1.1 Results
Let us consider two complex Hénon mappings $H_{i}:\left(\begin{array}{l}x \\ y\end{array}\right) \mapsto\left(\begin{array}{c}P_{i}(x)-a_{i} y \\ x\end{array}\right), i \in\{1,2\}$ together with their respective forward escaping sets $U_{1}^{+}, U_{2}^{+}$.

(c) The Author(s) 2017. This article is distributed under the terms of the Creative Commons Attribution 4.0 International License (http://creativecommons.org/licenses/by/4.0/), which permits unrestricted use, distribution, and reproduction in any medium, provided you give appropriate credit to the original author(s) and the source, provide a link to the Creative Commons license, and indicate if changes were made. 
Theorem 1.1 (Restrictions on degrees and Jacobians) Let $H_{1}$ and $H_{2}$ be two complex Hénon mappings of respective degrees $d_{1}, d_{2}$ and Jacobians $a_{1}, a_{2}$, such that the corresponding escaping sets are biholomorphic. Then one has the following:

(a) if one of the degrees $d_{1}, d_{2}$ is prime, then necessarily $d_{1}=d_{2}$;

(b) if $d_{1}=d_{2}$ (not necessarily prime), then the quotient $\left(\frac{a_{1}}{a_{2}}\right)$ is a $(d-1)$ th root of unity.

Quadratic case Observe that the theorem above already indicates that in degree 2 the Jacobian is prescribed. Actually, a more precise result can be obtained in this case:

Theorem 1.2 (Quadratic case) Let $H_{1}$ and $H_{2}$ be two quadratic Hénon maps. Then the following propositions are equivalent:

(a) the forward escaping sets are biholomorphic;

(b) $H_{1}=H_{2}$.

Ambiguity in higher degrees When the degree is at least 3, then the complex Hénon mapping is not completely determined by the analytic structure of the escaping set. There is a certain ambiguity in the determination that increases with the degree. Let us write

$$
H_{1}:\left(\begin{array}{l}
x \\
y
\end{array}\right) \mapsto\left(\begin{array}{c}
x^{3}+A_{1} x+B_{1}-a_{1} y \\
x
\end{array}\right) \quad \text { and } \quad H_{2}:\left(\begin{array}{l}
x \\
y
\end{array}\right) \mapsto\left(\begin{array}{c}
x^{3}+A_{2} x+B_{2}-a_{2} y \\
x
\end{array}\right)
$$

Theorem 1.3 (Cubic case) The forward escaping sets of $\mathrm{H}_{1}$ and $\mathrm{H}_{2}$ are biholomorphic if and only if one of the following conditions is realized: 1. $H_{1}$ and $H_{2} \quad$ are conjugate by $\quad\left(\begin{array}{l}x \\ y\end{array}\right) \mapsto\left(\begin{array}{l}-x \\ -y\end{array}\right)$ (equivalently
$\left.\left(a_{2}, A_{2}, B_{2}\right)=\left(a_{1}, A_{1},-B_{1}\right)\right)$;

2. $\left(a_{1}, A_{1}\right)=\left(a_{2},-A_{2}\right)$ and $B_{1}=B_{2}=0$, and in this case the two applications are not conjugate on $\mathbb{C}^{2}$, but their squares are conjugate by $g:\left(\begin{array}{l}x \\ y\end{array}\right) \mapsto\left(\begin{array}{c}i x \\ -i y\end{array}\right)$. Moreover, $g$ is an explicit isomorphism between the two escaping sets.

We notice that the Jacobian is still prescribed in the cubic case. Surprisingly, this is not necessarily true in higher degrees:

Theorem 1.4 (Jacobian non-preserved) The two maps $H_{1}:\left(\begin{array}{c}X \\ Y\end{array}\right) \mapsto\left(\begin{array}{c}X^{4}-Y \\ X\end{array}\right)$ and $H_{2}:\left(\begin{array}{c}X \\ Y\end{array}\right) \mapsto\left(\begin{array}{c}X^{4}-j Y \\ X\end{array}\right)$ where $j=e^{i \frac{2 \pi}{3}}$ have biholomorphic escaping sets, and an explicit isomorphism from $U_{2}^{+}$to $U_{1}^{+}$is given by

$$
\theta_{1}:\left(\begin{array}{c}
X \\
Y
\end{array}\right) \mapsto\left(\begin{array}{c}
\alpha X \\
\alpha^{7} Y
\end{array}\right), \quad \text { where } \alpha=e^{i \frac{2 \pi}{9}}
$$


In the last theorem, the map $\theta_{1}$ is actually a conjugacy on $\mathbb{C}^{2}$ between $H_{1}^{\circ 3}$ and $H_{2}^{\circ 3}$. Thus one can ask:

Conjecture Two complex Hénon mappings of degree d have biholomorphic escaping sets if and only if their $(d-1)$ th powers are conjugate.

\subsection{Idea of the proof}

The first ingredient is an explicit trivialization of the universal cover of the escaping set. Actually, we prefer to work in an intermediate cover described as follows:

Theorem 1.5 (Hubbard, Oberste-Vorth) There exists a cover $\widetilde{U}^{+}$of $U^{+}$, isomorphic to $(\mathbb{C}-\overline{\mathbb{D}}) \times \mathbb{C}$ in which the following Hénon mapping:

$$
H:\left(\begin{array}{l}
x \\
y
\end{array}\right) \mapsto\left(\begin{array}{c}
x^{d}+a_{d-2} x^{d-2}+\cdots+a_{0}-a y \\
x
\end{array}\right)
$$

has a lift written as

$$
\widetilde{H}:\left(\begin{array}{c}
\zeta \\
z
\end{array}\right) \mapsto\left(\begin{array}{c}
\zeta^{d} \\
\frac{a}{d} z+Q(\zeta)
\end{array}\right)
$$

where the polynomial $Q \in \mathbb{C}[X]$ is given by

(a) $Q(X)=X^{3}-\frac{a_{0}}{2} X$ ifd $=2$,

(b) $Q(X)=X^{d+1}-\frac{a_{d-2}}{d} X^{d-1}-\frac{a_{d-3}}{d} X^{d-2}-\left(\frac{a_{d-4}}{d}-\frac{a_{d-2}^{2}}{d^{2}}\right) X^{d-3}+\cdots$, if $d \geq 3$.

As such, this theorem is a rather straightforward generalization to any degree $d$ of a theorem due to Hubbard and Oberste-Vorth (compare [6] for the quadratic case). As these authors notice, we can obtain an explicit representation of the fundamental group as a group of biholomorphisms of $(\mathbb{C}-\overline{\mathbb{D}}) \times \mathbb{C}$ :

Theorem 1.6 (Hubbard, Oberste-Vorth) Let H be a complex Hénon mapping given by

$$
H:\left(\begin{array}{c}
x \\
y
\end{array}\right) \mapsto\left(\begin{array}{c}
P(x)-a y \\
x
\end{array}\right),
$$

where Pis of degree $d$ and let us call $\widetilde{H}$ the following lift of $H$ to $(\mathbb{C}-\overline{\mathbb{D}}) \times \mathbb{C}$ :

$$
\widetilde{H}:\left(\begin{array}{c}
\zeta \\
z
\end{array}\right) \mapsto\left(\begin{array}{c}
\zeta^{d} \\
\frac{a}{d} z+Q(\zeta)
\end{array}\right)
$$

where $Q$ is of degree $(d+1)$. Then we have the following:

(1) the fundamental group of $U^{+}$is isomorphic to $\mathbb{Z}\left[\frac{1}{d}\right]$;

(2) the element $\left[\frac{k}{d^{n}}\right] \quad$ of $\pi_{1}\left(U^{+}\right)$is represented by the biholomorphism

$$
\gamma_{\frac{k}{d^{n}}}:\left(\begin{array}{c}
\zeta \\
z
\end{array}\right) \mapsto\left(z+\frac{d}{a} \sum_{l=0}^{\infty}\left(\frac{d}{a}\right)^{l}\left(P\left(\zeta^{d^{l}}\right)-P\left(\left(e^{i 2 \pi \frac{k}{d^{n}}} \cdot \zeta\right.\right.\right.\right.
$$


The second ingredient of our proof is a construction used by Bousch [3]. Any biholomorphism between escaping sets that induces the identity on the fundamental groups can be lifted to an isomorphism $(\mathbb{C}-\overline{\mathbb{D}}) \times \mathbb{C}$. But these have a very simple form, and due to the very particular form of the fibers of $\pi: \widetilde{U^{+}} \rightarrow U^{+}$, it turns out that very few of these isomorphisms can satisfy the additional requirement of sending the fibers of $\pi_{1}$ to the fibers of $\pi_{2}: \widetilde{U_{2}^{+}} \rightarrow U_{2}^{+}$. The rigidity derives exactly from this fact.

\section{Equality of degrees}

Our objective is the following:

Theorem 2.1 (Conditions on degrees) Let $U_{1}^{+}$be the escaping set of a complex Hénon mapping of degree $d$, with $d$ being a prime integer. Assume that the escaping set $U_{2}^{+}$of some other Hénon mapping is biholomorphic to $U_{1}^{+}$; then necessarily the polynomials $P_{1}, P_{2}$ have the same degree $d$.

Let us prove first an easy lemma:

Lemma 2.2 The degree d'of $\mathrm{H}_{2}$ is a power of $d$.

Proof Let $\theta: U_{1}^{+} \rightarrow U_{2}^{+}$be the biholomorphism. The map $\psi=\theta^{-1} \circ H_{2} \circ \theta: U_{1}^{+} \rightarrow U_{1}^{+}$induces an automorphism on the fundamental group of $U_{1}^{+}=\mathbb{Z}\left[\frac{1}{d}\right]$. These automorphisms are the multiplications by an invertible element of $\mathbb{Z}\left[\frac{1}{d}\right]$. Therefore, $\psi$ induces the multiplication by a power of $d$. Since $H_{2}$ induces the multiplication by $d^{\prime}$ and all the induced maps $\theta_{\star}, \theta_{\star}^{-1}$ commute (being multiplications by some integer), we deduce that $d^{\prime}=d^{n}$ for some integer $n$.

Lemma 2.3 There exists a biholomorphism between the escaping sets that induces the identity on the fundamental groups.

Proof Since $d^{\prime}=d^{n}$, we deduce that $\mathbb{Z}\left[1 / d^{\prime}\right]=\mathbb{Z}[1 / d]$. Therefore, the biholomorphism $\theta$ must induce an automorphism of $\mathbb{Z}[1 / d]$. These are made of multiplication by a power of $d$. By precomposing $\theta$ with an iterate of $H_{1}$, we can assume that $\theta$ induces $\pm I d$ on $\mathbb{Z}[1 / d]$. Now we notice that the projection $\pi:(\mathbb{C}-\overline{\mathbb{D}}) \times \mathbb{C} \rightarrow U^{+}$induces on the fundamental groups the map $\pi_{\star}: \mathbb{Z} \mapsto \mathbb{Z} \subset \mathbb{Z}[1 / d]$. Therefore, by standard covering theory $\theta$ can be lifted to a self-map of $(\mathbb{C}-\overline{\mathbb{D}}) \times \mathbb{C}$. If needed, one can postcompose with a deck transformation and assume that the lifted map $\tilde{\theta}$ is actually an isomorphism of $(\mathbb{C}-\overline{\mathbb{D}}) \times \mathbb{C}$.

Lemma 2.4 The isomorphisms of $(\mathbb{C}-\overline{\mathbb{D}}) \times \mathbb{C}$ are given by
$\phi:\left(\begin{array}{c}\zeta \\ z\end{array}\right) \mapsto\left(\begin{array}{c}\alpha \zeta \\ \alpha(\zeta) z+\beta(\zeta)\end{array}\right)$, where $\alpha \in \mathbb{C}$ and $\alpha(\zeta), \beta(\zeta)$ are two holomorphicfunctions. Proof Left to the reader. See Bousch's thesis [3] for details. 
This implies, as Bousch notices, that $\widetilde{\theta}$ actually induces $+I d$, because a loop $\left(\begin{array}{c}R \cdot \mathrm{e}^{i 2 \pi t} \\ 0\end{array}\right)$ in $(\mathbb{C}-\overline{\mathbb{D}}) \times \mathbb{C}$ descends to the generator +1 of $\pi_{1}\left(U^{+}\right)$in $U_{1}^{+}$and is sent to the loop $\left(\begin{array}{c}\alpha R \cdot \mathrm{e}^{i 2 \pi t} \\ \beta\left(R \cdot \mathrm{e}^{i 2 \pi t}\right)\end{array}\right)$ which descends to the same generator in $U^{+}$.

\subsection{Lifting of biholomorphisms}

Let us prove now that the degree $d^{\prime}$ must be equal to $d$ itself. At this point, we have the following situation:

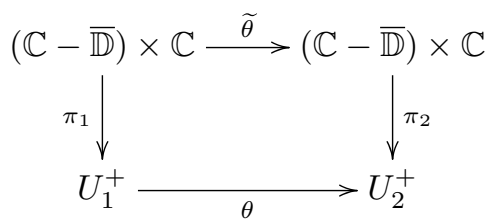

where $\widetilde{\theta}:\left(\begin{array}{c}\zeta \\ z\end{array}\right) \mapsto\left(\begin{array}{c}\alpha \zeta \\ \alpha(\zeta) z+\beta(\zeta)\end{array}\right)$.

\subsection{Fibers of $\pi: \widetilde{U}^{+} \rightarrow U^{+}$are mapped to fibers} Theorem 2.5 (Hubbard, Oberste-Vorth) Let $H:\left(\begin{array}{l}x \\ y\end{array}\right) \mapsto\left(\begin{array}{c}P(x)-\text { ay } \\ x\end{array}\right)$ where $P$ is of
degree d. If a lift of $H$ to $(\mathbb{C}-\overline{\mathbb{D}}) \times \mathbb{C}$ is given by

$$
\widetilde{H}:\left(\begin{array}{c}
\zeta \\
z
\end{array}\right) \mapsto\left(\begin{array}{c}
\zeta^{d} \\
\frac{a}{d} z+Q(\zeta)
\end{array}\right)
$$

where $Q$ is a monic polynomial of degree $d+1$, then the element $\left[\frac{k}{d^{n}}\right]$ of $\pi_{1}\left(U^{+}\right)$is represented by this biholomorphism:

$$
\gamma_{\frac{k}{d^{n}}}:\left(\begin{array}{c}
\zeta \\
z
\end{array}\right) \mapsto\left(z+\frac{d}{a} \sum_{l=0}^{\infty}\left(\frac{d}{a}\right)^{l}\left(Q\left(\zeta^{e^{l}}\right)-Q\left(\left(e^{i 2 \pi \frac{k}{d^{n}}} \cdot \zeta\right)^{d^{l}}\right)\right) .\right.
$$

Proof Once we know $\gamma_{\frac{1}{d^{n}}}$, we can easily deduce the expression of $\gamma_{\frac{-1}{d^{n}}}$, and then the expression of all its iterates, in other words of all the other elements. Let us prove the result for $\gamma_{\frac{1}{d^{n}}}$ by induction on $n$ :

- for $n=1$, we get the identity;

- then we notice that $\widetilde{H} \circ \gamma_{\frac{1}{d^{n+1}}}=\gamma_{\frac{1}{d^{n}}} \circ \widetilde{H}$. Hence

$$
z^{\prime \prime}=\frac{a}{d} z^{\prime}+Q\left(\mathrm{e}^{i \frac{2 \pi}{d^{n+1}}} \cdot \zeta\right)
$$

and also

$$
z^{\prime \prime}=\frac{a}{d} z+Q(\zeta)+\frac{d}{a} \sum_{l=0}^{\infty}\left(\frac{d}{a}\right)^{l}\left(Q\left(\zeta^{d^{l}}\right)-Q\left(\left(\mathrm{e}^{i 2 \pi \frac{1}{d^{n}} \zeta}\right)^{d^{l}}\right)\right)
$$




$$
\frac{a}{d} z^{\prime}+Q\left(\mathrm{e}^{i \frac{2 \pi}{d^{n+1}}} \cdot \zeta\right)=\frac{a}{d} z+Q(\zeta)+\frac{d}{a} \sum_{l=0}^{\infty}\left(\frac{d}{a}\right)^{l}\left(Q\left(\zeta^{d \cdot d^{l}}\right)-Q\left(\left(\mathrm{e}^{i 2 \pi \frac{1}{d^{n}}} \zeta^{d}\right)^{d^{l}}\right)\right)
$$

Conservation of fibers This whole section is an application of Bousch's procedure.

Let us express the condition that $\widetilde{\theta}$ must send the fiber of $\pi_{1}$ above a point $p \in U^{+}$ on the fiber of $\pi_{2}$ above $\theta(p)$. The fiber $\mathcal{F}_{p}$ above $p \in U_{1}^{+}$. Let $H_{1}$ be the Hénon mapping of Jacobian $a_{1}$, and the corresponding polynomial $P(X)=X^{d_{1}}+A_{d_{1}-2}^{(1)} X^{d_{1}-2}+\cdots A_{0}^{(1)}$. Let $\tilde{p}=\left(\begin{array}{l}\zeta \\ z\end{array}\right)$ be a point in the fiber of $p \in U_{1}^{+}$. The other points in the fiber are

$$
\left(z+\frac{d_{1}}{a_{1}} \sum_{l=0}^{\infty}\left(\frac{d_{1}}{a_{1}}\right)^{l}\left[\mathrm{e}^{i \frac{2 k \pi}{d_{1}^{n}}} \zeta \zeta^{d_{1}^{l}}\right)-Q_{1}\left(\left(\mathrm{e}^{\left.\left.\left.i \frac{2 k \pi}{d_{1}^{n}} \zeta\right)^{d_{1}^{l}}\right)\right]}\right)\right.\right.
$$

where $Q_{1}(\zeta)=\zeta^{d_{1}+1}-\frac{A_{d_{1}-2}^{(1)}}{d_{1}} \zeta^{d_{1}-1}-\cdots-\frac{A_{0}^{(1)}}{d_{1}}$.

Points having the same projection as $\tilde{\theta}(\tilde{p})$. Since we have

$$
\tilde{\theta}:\left(\begin{array}{c}
\zeta \\
z
\end{array}\right) \mapsto\left(\begin{array}{c}
\alpha \zeta \\
\alpha(\zeta) z+\beta(\zeta)
\end{array}\right)
$$

we deduce that the other points having the same projection by $\pi_{2}$ as the point $\tilde{\theta}(\tilde{p})$ are the following (for readability reasons, we write $d_{2}$ instead of $d^{\prime}$, which is actually a power $d^{k}$ of $\left.d\right)$ :

$$
\left.\left(\alpha \cdot \mathrm{e}^{i \frac{2 s \pi}{d_{2}^{q}}} \zeta, \alpha(\zeta) z+\beta(\zeta)+\frac{d_{2}}{a_{2}} \sum_{l=0}^{\infty}\left(\frac{d_{2}}{a_{2}}\right)^{l}\left[Q_{2}\left((\alpha \zeta)^{d_{2}^{l}}\right)-Q_{2}\left(\left(\mathrm{e}^{i \frac{2 s \pi}{d_{2}^{q}}} \alpha \zeta\right)^{d_{2}^{l}}\right)\right)\right]\right)
$$

where $Q_{2}$ is a polynomial of degree $d_{2}+1$. Notice that the sums involved are actually finite sums. The image by $\tilde{\theta}$ of the fiber $\mathcal{F}_{p}$. This image is made of all the points with the first coordinate $\left(\alpha \cdot \mathrm{e}^{i \frac{2 k \pi}{d_{1}^{n}}} \zeta\right)$ and the second coordinate

$$
\alpha\left(\mathrm{e}^{i \frac{2 k \pi}{d_{1}^{n}}} \zeta\right) \cdot\left[z+\frac{d_{1}}{a_{1}} \sum_{l=0}^{\infty}\left(\frac{d_{1}}{a_{1}}\right)^{l}\left(Q_{1}\left(\zeta^{d_{1}^{l}}\right)-Q_{1}\left(\left(\mathrm{e}^{i \frac{2 k \pi}{d_{1}^{n}}} \zeta\right)^{d_{1}^{l}}\right)\right)\right]+\beta\left(\mathrm{e}^{i \frac{2 k \pi}{d_{1}^{n}}} \zeta\right)
$$

Now the remarkable insight of Bousch is that the comparison of these fibers yields all the information we need. Namely we get: comparison of the two fibers.

Lemma 2.6 The degree $d_{2}=d^{\prime}=d^{t}$ is equal to $d$.

Proof Fix $z=0$ and take a fixed $\zeta$. Fix also $k=1$ and see what happens for the particular choice of integers $n=t \cdot b$, with $b$ going to infinity. It turns out that all the terms $\alpha\left(\mathrm{e}^{i \cdot \frac{2 \pi}{d_{1}^{n}}} \cdot \zeta\right), \beta\left(\mathrm{e}^{i \cdot \frac{2 \pi}{d_{1}^{n}}} \cdot \zeta\right)$ remain bounded, whereas the rest grows like the leading terms in the sum which are $\zeta^{\left(d_{2}+1\right) \cdot d_{2}^{b-1}}=\zeta^{\left(d^{t}+1\right) \cdot d^{t \cdot(b-1)}}$ in one case, and $\zeta^{(d+1) \cdot d^{b t-1}}$ in 
the other. This implies that $t$ must be equal to one, and therefore $d_{2}=d^{t}=d_{1}$, which is what we wanted.

Summary At this point, we have proved that if an escaping set of a Hénon map with prime degree $d$ is biholomorphic to another escaping set, then necessarily the other Hénon map has degree $d$ as well.

\subsection{Condition on the Jacobians}

Now that we have an explicit description of the fibers, one can write down necessary condition for them to coincide.

Theorem 2.7 (Condition on the Jacobians) If $U_{1}^{+}$and $U_{2}^{+}$are biholomorphic, then $\left(\frac{a_{1}}{a_{2}}\right)$ is $a(d-1)$ th root of the unity, where $d$ is the common degree of both maps.

Proof We fix $k=1$ and let $n$ vary: one can first deduce that $\alpha\left(\mathrm{e}^{i \frac{2 k \pi}{d^{n}}} \zeta\right)=\alpha(\zeta)$, for any $\mathrm{n}$, any $\zeta$. Therefore, the function $\alpha$ is a constant $K$ (a non-zero constant because $\widetilde{\theta}$ is an isomorphism): $\forall \zeta \in(\mathbb{C}-\overline{\mathbb{D}}), \alpha(\zeta)=K$. Now fix $\zeta$ and let $n$ vary. The leading terms of the two

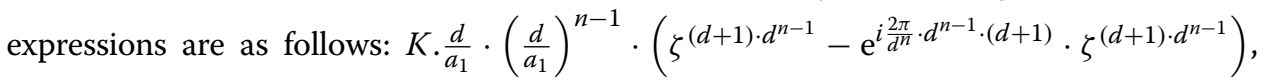
for the first one, and $\frac{d}{a_{2}} \cdot\left(\frac{d}{a_{2}}\right)^{n-1} \cdot \alpha^{(d+1) \cdot d^{n-1}} \cdot \zeta^{(d+1) \cdot d^{n-1}}\left(1-\mathrm{e}^{i \frac{2 \pi}{d}}\right)$, for the second one.

Notice that the terms $\beta(\zeta)$ and $\beta\left(\mathrm{e}^{i \frac{2 \pi}{d^{n}}} \zeta\right)$ are bounded. After comparing these leading terms, we get for $n$ large enough $\lim _{n}\left(\left(\frac{a_{1}}{a_{2}}\right)^{n} \alpha^{(d+1) \cdot d^{n-1}}\right)=K$.

Similarly, we obtain $\lim _{n}\left(\left(\frac{a_{1}}{a_{2}}\right)^{n+1} \alpha^{(d+1) \cdot d^{n}}\right)=K$. Hence, by quotienting $\lim _{n}\left(\left(\frac{a_{1}}{a_{2}}\right) \alpha^{(d+1) \cdot(d-1) \cdot d^{n-1}}\right)=1$.

By quotienting again one finally gets $\lim _{n}\left(\alpha^{(d+1) \cdot(d-1)^{2} \cdot d^{n-1}}\right)=1$.

But this implies $\left(\frac{a_{1}}{a_{2}}\right)^{d-1}=1$.

\subsection{Quadratic case}

We recall the normalized form in the quadratic case:

$$
H_{a, c}:\left(\begin{array}{l}
x \\
y
\end{array}\right) \mapsto\left(\begin{array}{c}
x^{2}+c-a y \\
x
\end{array}\right)
$$

Theorem 2.8 Let $U_{1}^{+}$and $U_{2}^{+}$be the escaping sets of two quadratic complex Hénon mappings written in normalized form, $H_{a, c}$ and $H_{a^{\prime}, c^{\prime}}$,respectively. If $U_{1}^{+}$and $U_{2}^{+}$are biholomorphic then $a=a^{\prime}$ and $c=c^{\prime}$.

Proof Since we know from the preceding theorem that the Jacobians are equal, we can pursue the comparison of the fibers by looking at the next leading term.

For $\zeta$ fixed, $k=1$ and $n$ going to infinity, we have

$$
K\left(\frac{2}{a}\right)^{n}\left(\frac{-c}{2}\right) \zeta^{2^{n-1}}\left(1-\mathrm{e}^{-i \frac{2 \pi}{2}}\right) \sim\left(\frac{2}{a^{\prime}}\right)^{n}\left(\frac{-c^{\prime}}{2}\right) \alpha^{2^{n-1}} \zeta^{2^{n-1}}\left(1-\mathrm{e}^{-i \frac{2 \pi}{2}}\right) .
$$


But we already knew $K\left(\frac{2}{a}\right)^{n} \zeta^{3.2^{n-1}}\left(1-\mathrm{e}^{i \frac{2 \pi}{2}}\right) \sim\left(\frac{2}{a^{\prime}}\right)^{n} \alpha^{3.2^{n-1}} \zeta^{3.2^{n-1}}\left(1-\mathrm{e}^{-i \frac{2 \pi}{2}}\right)$.

Therefore $c \sim c^{\prime} \cdot \alpha^{2^{n}}$ which implies $c=c^{\prime}$.

\subsection{Cubic case}

This case is interesting because the degree is low enough to enable us to compute everything precisely, and at the same time it is the first case for which the analytic structure of the escaping set does not completely determine the Hénon map that created it.

We recall that if $H_{1}\left(\begin{array}{l}x \\ y\end{array}\right)=\left(\begin{array}{c}x^{3}+A x+B-a y \\ x\end{array}\right)$, then a lift of $H_{1}$ is

$$
\widetilde{H}_{1}\left(\begin{array}{l}
\zeta \\
z
\end{array}\right)=\left(\begin{array}{c}
\zeta^{3} \\
\frac{a}{3} z+\zeta^{4}-\frac{A}{3} \zeta^{2}-\frac{B}{3} \zeta
\end{array}\right)
$$

Theorem 2.9 (Cubic case) The forward escaping sets $U_{1}^{+}$and $U_{2}^{+}$of the two cubic Hénon mappings are biholomorphic if and only if one of the following conditions is realized:

1. $H_{1} \quad$ and $H_{2} \quad$ are conjugate by $\left(\begin{array}{l}x \\ y\end{array}\right) \mapsto\left(\begin{array}{l}-x \\ -y\end{array}\right) \quad$ (equivalently $\left(a^{\prime}, A^{\prime}, B^{\prime}\right)$
$=(a, A,-B)) ;$ 2. $(a, A)=\left(a^{\prime},-A^{\prime}\right) \quad$ and $B=B^{\prime}=0, \quad$ and in this case the two applications are not conjugate on $\mathbb{C}^{2}, \quad$ but their squares are conjugate by $\left(\begin{array}{c}x \\ y\end{array}\right) \mapsto\left(\begin{array}{c}i x \\ -i y\end{array}\right)$. Moreover, this map is an explicit isomorphism between the two escaping sets.

Remark 1 A necessary condition for the escaping sets to be biholomorphic is that the Jacobians are equal. We will see that this is not true in degrees higher than 4 .

Proof Again we compare the successive leading terms:

First leading term.

$$
K \cdot\left(\frac{3}{a}\right)^{n} \cdot\left(1-\mathrm{e}^{i \frac{2 \pi}{3}}\right) \cdot \zeta^{4 \cdot 3^{n-1}} \sim\left(\frac{3}{a^{\prime}}\right)^{n} \cdot\left(1-\mathrm{e}^{i \frac{2 \pi}{3}}\right) \cdot \alpha^{4 \cdot 3^{n-1}} \zeta^{4 \cdot 3^{n-1}} \cdot
$$

Second leading term.

$$
K \cdot\left(\frac{3}{a}\right)^{n} \cdot\left(1-\mathrm{e}^{-i \frac{2 \pi}{3}}\right) \cdot\left(\frac{-A}{3}\right) \zeta^{2 \cdot 3^{n-1}} \sim\left(\frac{3}{a^{\prime}}\right)^{n} \cdot\left(1-\mathrm{e}^{-i \frac{2 \pi}{3}}\right) \cdot\left(\frac{-A^{\prime}}{3}\right) \cdot \alpha^{2 \cdot 3^{n-1}} \zeta^{2 \cdot 3^{n-1}} .
$$

\section{Third leading term.}

$$
K \cdot\left(\frac{3}{a}\right)^{n} \cdot\left(1-\mathrm{e}^{-i \frac{4 \pi}{3}}\right) \cdot\left(\frac{-B}{3}\right) \zeta^{3^{n-1}} \sim\left(\frac{3}{a^{\prime}}\right)^{n} \cdot\left(1-\mathrm{e}^{-i \frac{4 \pi}{3}}\right) \cdot\left(\frac{-B^{\prime}}{3}\right) \cdot \alpha^{3^{n-1}} \zeta^{3^{n-1}} .
$$

From the two first leading terms, we get $\alpha^{2.3^{n-1}} A^{\prime} \sim A$, which yields thanks to the second relation $K \cdot\left(\frac{3}{a}\right)^{n} \sim\left(\frac{3}{a^{\prime}}\right)^{n}$ and thus (thanks to the first relation) $\alpha^{4.3^{n}}=1$ for $n$ large enough, and we also have that $a=a^{\prime}$ and $K=1$.

Knowing that $\alpha^{2.3^{n-1}} A^{\prime} \sim A$ and $\alpha^{4.3^{n-1}}=1$ for $n$ large enough, we deduce that $\alpha^{2.3^{n-1}}= \pm 1$ and also that $A= \pm A^{\prime}$. Let us discuss those cases:

- $\mathrm{A}=\mathrm{A}^{\prime}$ We already know that $B \sim B^{\prime} \alpha^{3^{n}}$ and also that $\alpha^{4.3^{n}} \sim 1$ for $n$ large enough. Therefore, either $B=B^{\prime}=0$, or $B$ and $B^{\prime}$ are non-zero and satisfy $B= \pm B^{\prime}$. 
- $\mathrm{A}=-\mathrm{A}^{\prime}$ Similarly, either $B=B^{\prime}=0$, or $B$ and $B^{\prime}$ are both non-zero, but then they must satisfy $\pm i=\frac{B}{B^{\prime}}=\alpha^{3^{n}}$, which is impossible because $\pm i^{3^{n}}$ is not constant.

It remains to decide which are the cases that give non-trivial isomorphisms between the escaping sets. Let us show that in the case $(a, A)=\left(a^{\prime},-A^{\prime}\right)$ and $B=B^{\prime}=0$ the two maps are not conjugate (unless $A=A^{\prime}$ ): notice that each map has three fixed points, two of them having the same Jacobian matrix. Indeed, the fixed points are solutions of $X^{3}+A X-a X=X$ for $H_{1}$ and the Jacobian matrix at the two fixed points $\pm \sqrt{A-a-1}$ is

$$
\left(\begin{array}{cc}
4 A-3 a-3 & -a \\
1 & 0
\end{array}\right)
$$

whereas for the second map two fixed points have a common Jacobian matrix equal to

$$
\left(\begin{array}{cc}
-4 A-3 a-3 & -a \\
1 & 0
\end{array}\right)
$$

The traces being distinct, no conjugacy can send these two fixed points on the two other ones.

\subsection{In degree $3, U^{+}$does not determine $H$}

In this section we exhibit explicit examples of pairs of different maps having biholomorphic escaping sets.

Theorem $\quad 2.10$ Let $\quad$ us $\quad$ consider $\quad H_{1}:\left(\begin{array}{c}X \\ Y\end{array}\right) \mapsto\left(\begin{array}{c}X^{3}+C X-a Y \\ X\end{array}\right)$ and $H_{2}:\left(\begin{array}{c}X \\ Y\end{array}\right) \mapsto\left(\begin{array}{c}X^{3}-C X-a Y \\ X\end{array}\right)$. Then the map $\theta:\left(\begin{array}{c}X \\ Y\end{array}\right) \mapsto\left(\begin{array}{c}i X \\ -i Y\end{array}\right)$ is an isomorphism of $\mathbb{C}^{2}$ into itself that restricts to an isomorphism from $U_{2}^{+}$into $U_{1}^{+}$. Moreover, $\theta$ conjugates the squares of the two maps.

- Proof First notice that $-I d$ commutes with $H_{1}$ (and also $H_{2}$ ):

$$
\left(\begin{array}{c}
X \\
Y
\end{array}\right) \mapsto\left(\begin{array}{c}
-X \\
-Y
\end{array}\right) \mapsto\left(\begin{array}{c}
-X^{3}-C X+a Y \\
-X
\end{array}\right) \mapsto\left(\begin{array}{c}
X^{3}+C X-a Y \\
X
\end{array}\right) \text {. }
$$

and that $\theta^{\circ 2}=-I d$

- Then one verifies

$\theta^{-1} \circ H_{1}=H_{2} \circ \theta ;$

indeed

$$
\theta^{-1} \circ H_{1}:\left(\begin{array}{c}
X \\
Y
\end{array}\right) \mapsto\left(\begin{array}{c}
X^{3}+C X-a Y \\
X
\end{array}\right) \mapsto\left(\begin{array}{c}
-i X^{3}-i C X+a i Y \\
i X
\end{array}\right)
$$

and

$$
H_{2} \circ \theta:\left(\begin{array}{c}
X \\
Y
\end{array}\right) \mapsto\left(\begin{array}{c}
i X \\
-i Y
\end{array}\right) \mapsto\left(\begin{array}{c}
-i X^{3}-i C X+a i Y \\
i X
\end{array}\right) .
$$


Therefore, one can deduce that $\theta^{-1} \circ H_{1} \circ \theta=H_{2} \circ \theta^{\circ 2}=H_{2} \circ(-I d)=-I d \circ H_{2}$. By iterating the relation, one gets $\theta^{-1} \circ H_{1}^{\circ n} \circ \theta=(-I d)^{n} \circ H_{2}^{\circ n}$.

To conclude, let $p \in U_{2}^{+}$; then $\left\|(-I d)^{n} \circ H_{2}^{\circ n}(p)\right\|$ is unbounded but then $\theta^{-1} \circ H_{1}^{\circ n}(\theta(p))$ is unbounded and then $\theta(p) \in U_{1}^{+}$. It remains to show that $\theta$ conjugates the squares of the maps: start from $H_{1}=\theta \circ H_{2} \circ \theta$ and deduce $H_{1}^{2}=\theta \circ H_{2} \circ(-I d) \circ H_{2} \circ \theta$, or if one prefers, by remembering that $-I d$ commutes with $H_{i}$ and that $-I d \circ \theta=\theta^{-1}$, one obtains $H_{1}^{2}=\theta^{-1} \circ H_{2}^{2} \circ \theta$.

\section{Jacobian not prescribed}

Let us show with an explicit example that in degree 4 the Jacobian is not necessarily prescribed by the analytic structure of the escaping set.

Theorem 3.1 (Jacobian non-preserved) The following maps:

- $H_{1}:\left(\begin{array}{l}x \\ y\end{array}\right) \mapsto\left(\begin{array}{c}x^{4}-y \\ x\end{array}\right)$

- $H_{2}:\left(\begin{array}{l}x \\ y\end{array}\right) \mapsto\left(\begin{array}{c}x^{4}-j y \\ x\end{array}\right)$, where $j=e^{i \frac{2 \pi}{3}}$,

have biholomorphic escaping sets, and an explicit isomorphism from $U_{2}^{+}$to $U_{1}^{+}$is given by $\theta_{1}:\left(\begin{array}{c}x \\ y\end{array}\right) \mapsto\left(\begin{array}{c}\alpha x \\ \alpha^{7} y\end{array}\right)$, where $\alpha=e^{i \frac{2 \pi}{9}}$.

Proof Let us introduce the linear map $L:\left(\begin{array}{l}x \\ y\end{array}\right) \mapsto\left(\begin{array}{c}j^{2} x \\ j^{2} y\end{array}\right)$. It commutes with $H_{1}$ and $H_{2}$ since $L \circ H_{1}:\left(\begin{array}{l}x \\ y\end{array}\right) \mapsto\left(\begin{array}{c}j^{2} x^{4}-j^{2} y \\ j^{2} x\end{array}\right)=\left(\begin{array}{c}j^{8} x^{4}-j^{2} y \\ j^{2} x\end{array}\right)=H_{1} \circ L\left(\begin{array}{l}x \\ y\end{array}\right)$, and

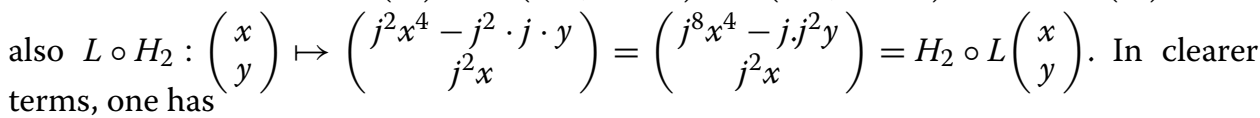

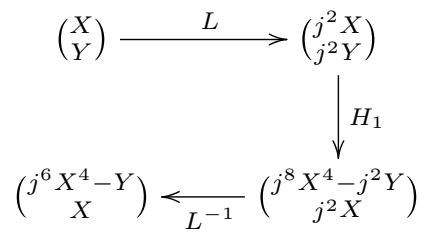

Now the key property is that $H_{1}$ and $H_{2}$ are almost conjugate: more precisely, let us write $\theta_{2}:\left(\begin{array}{c}X \\ Y\end{array}\right) \mapsto\left(\begin{array}{c}\alpha^{5} X \\ \alpha^{8} Y\end{array}\right)$; then the following diagram commutes:

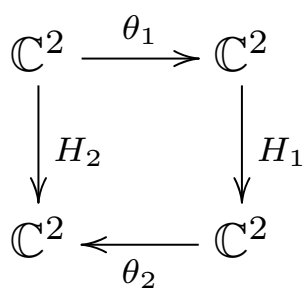

Indeed, we have 


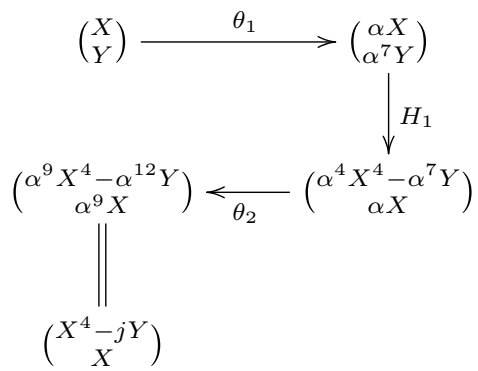

Therefore we proved $H_{2}=\theta_{2} \circ H_{1} \circ \theta_{1}$

By iterating further, one gets

$$
H_{2}^{\circ n}=\theta_{2} \circ H_{1} \circ\left(\theta_{1} \circ \theta_{2}\right) \circ H_{1} \circ\left(\theta_{1} \circ \theta_{2}\right) \ldots\left(\theta_{1} \circ \theta_{2}\right) \circ H_{1} \circ \theta_{1}
$$

but one also has $\theta_{1} \circ \theta_{2}\left(\begin{array}{c}X \\ Y\end{array}\right)=\left(\begin{array}{c}\alpha^{6} X \\ \alpha^{15} Y\end{array}\right)=\left(\begin{array}{c}j^{2} X \\ j^{2} Y\end{array}\right)=L\left(\begin{array}{c}X \\ Y\end{array}\right)$.

If one recalls that $L$ commutes with both $H_{1}, H_{2}$, one deduces that $H_{2}^{\circ n} \circ \theta_{1}^{-1}=\theta_{2} \circ L^{n} \circ H_{1}^{\circ n}$.

Precomposing by $L$ or $\theta_{i}$ does not change the norm of a vector (each component being multiplied by a complex number on the unit circle). Therefore, if $p \in U_{1}^{+}$then $\left\|H_{1}^{\circ n}(p)\right\|$ is unbounded and also $\| H_{2}^{\circ n}\left(\theta_{1}^{-1}(p) \|\right.$, but this shows that $\theta_{1}^{-1}(p)$ belongs to $U_{2}^{+}$.

Authors' contributions

SB wrote the first version, and all the authors discussed the topic and obtained the results. All authors read and approved the final manuscript.

\section{Author details}

${ }^{1}$ Mathematics and Statistics Institute, University of Sao Paulo, Rua do Matão 1010, São Paulo 05508-090, Brazil. ${ }^{2}$ Department of Mathematics, Stony Brook University, Stony Brook, NY 11794-3651, USA.

\section{Acknowledgements}

The first author wishes to acknowledge support from the FAPESP Grants 2013/23643-4 and 2011/16265-8.

\section{Competing interests}

The authors declare that they have no competing interests.

\section{Appendix}

This section gathers the more technical parts, which are essentially convenient change of coordinates within the escaping sets. We provide a bit more material than is strictly necessary, in the hope that such calculations might be useful for further investigations.

\section{Uniformizations}

Theorem 4.1 In the coordinates $(\zeta, z)$, the Hénon map is given by

$$
H(\zeta, z)=\left(\begin{array}{c}
\zeta^{d} \\
\frac{a}{d} z+Q(\zeta)
\end{array}\right)
$$


where the degree $(d+1)$ polynomial (without constant term) $Q$ is as follows:

(1) Degree 2: Given that $P(x)=x^{2}+$ cone has $Q(\zeta)=\zeta^{3}-\frac{c}{2} \zeta$.

(2) Degree 3: For $P(x)=x^{3}+A x+B$ one has $Q(\zeta)=\zeta^{4}-\frac{A}{3} \zeta^{2}-\frac{B}{3} \zeta$.

(3) Degree 4: For $P(x)=x^{4}+A x^{2}+B x+C$, one has

$$
Q(\zeta)=\zeta^{5}-\frac{A}{4} \zeta^{3}-\frac{B}{4} \zeta^{2}+\frac{A^{2}-8 C}{32} \zeta
$$

(4) Degree 5: For $P(x)=x^{5}+A x^{3}+B x^{2}+C x+D$, one has

$$
Q(\zeta)=\zeta^{6}-\frac{A}{5} \zeta^{4}-\frac{B}{5} \zeta^{3}+\frac{A^{2}-5 C}{25} \zeta^{2}-\frac{3 A B+5 D}{25} \zeta
$$

(5) Degree 6: For $P(x)=x^{6}+A x^{4}+B x^{3}+C x^{2}+D x+E$, one has

$$
Q(\zeta)=\zeta^{7}-\frac{A}{6} \zeta^{5}-\frac{B}{6} \zeta^{4}+\frac{A^{2}-4 C}{24} \zeta^{3}+\frac{A B-3 D}{18} \zeta^{2}+\alpha \cdot \zeta
$$

where the coefficient $\alpha$ is given by

$$
-\frac{2 A^{3}}{6^{3}}-\frac{B^{2}}{18}-\frac{12 A C-5 A^{3}}{108}-\frac{E}{6}+\frac{5 B^{2}}{72} \frac{10 A C}{72}-\frac{55 A^{3}}{1296} .
$$

\section{Second coordinate $z(x, y)$}

Lemma 4.2 One can find two constants $M>0, R>0$ for which there exists on the domain

$$
M^{+}:=\left\{\left(\begin{array}{c}
x \\
y
\end{array}\right) \in V^{+} ;\left|\phi^{+}\left(\begin{array}{c}
x \\
y
\end{array}\right)\right|>M \max \{R,|y|\}\right\}
$$

an analytic function $\psi$ satisfying

$$
\psi(H(x, y))=\frac{a}{d} \psi(x, y)+Q(\zeta(x, y))
$$

where $Q$ is a polynomial function of degree $d+1$.

Proof Following [9], one introduces $\psi(x, y)=\zeta \int_{0}^{x(\zeta, y)} \frac{\partial x}{\partial \zeta}(\zeta, u) \mathrm{d} u$ and then expresses the Hénon map in the coordinates $(\zeta, \psi)$.

Lemma 4.3 The function $Q(\zeta)$ is the polynomial part of the power series

$$
S(\zeta)=\zeta^{d} \int_{0}^{x(\zeta, 0)} \frac{\partial x}{\partial \zeta}\left(\zeta^{d}, u\right) d u
$$

Moreover, if the Jacobian $a$ is not equal to the degree $d$, one can assume that $Q$ has no constant term by performing a further change of variables $\psi(z) \mapsto \psi(z)+\alpha$ (where $\alpha$ is a constant). 
Proof This is proved in [9], page 248.

Study of $\zeta=\phi^{+}(x, y)$. Near the point $p=[1: 0: 0] \in \mathbb{C P}^{2}$, we use the local coordinates $(t, w)=(1 / x, y / x)$.

Lemma 4.4 Near the point $p$, the change of variables $\left(\begin{array}{c}t \\ w\end{array}\right) \mapsto\left(\begin{array}{c}1 / \zeta \\ y / \zeta\end{array}\right)$ is a biholomorphism that is tangent to the identity.

Proof Favre [4] proved that $1 / \zeta$ can be expressed as a power series in $(t, w)$ of the form $t+R_{2}(t, w)$ where $R_{2}$ has monomials with total degree in $t, w$ that is larger than or equal to 2 . For the second coordinate, just observe that $y / \zeta=(y / x) \cdot(x / \zeta)=w \cdot(1+R(t, w))$, where all monomials in $R$ have total degree at least 1.

Immediately, this provides useful expressions for $x(\zeta, y)$ and $\zeta(x, y)$.

For the next few lemmas we will work in the coordinates $t, w$ to simplify, as it is easier to deal with power series rather than two-dimensional Laurent series.

Lemma 4.5 Using the variables $t=1 / x$ and $w=y / x$, the function $1 / \zeta$ has the following expansion in a neighborhood of the point $p=[1: 0: 0]$ :

$$
\frac{1}{\zeta}=t(1+t \alpha(t, w))
$$

where $\alpha(t, w)$ is a power series in $t, w$ converging near $p$.

Proof We recall the expression of the Hénon map in the variables $t, w$ :

$$
H(t, w)=\left(\begin{array}{c}
\frac{t^{d}}{t^{d} P(1 / t)-a w t^{d-1}} \\
\frac{t^{d}-1}{t^{d} P(1 / t)-a w t^{d-1}}
\end{array}\right)
$$

Now, if we write $t_{n}(t, w)$ the first coordinate of $H^{n}(t, w)$ we have $1 / \zeta=\lim \left(t_{n}\right)^{1 / d^{n}}$. More precisely, $1 / \zeta$ is obtained as the limit of the infinite product:

$$
1 / \zeta=t \cdot\left(\frac{\frac{t^{d}}{t^{d} P(1 / t)-a w t^{d-1}}}{t^{d}}\right)^{1 / d} \cdot\left(\frac{\frac{t_{1}^{d}}{t_{1}^{d} P\left(1 / t_{1}\right)-a w_{1} t_{1}^{d-1}}}{t_{1}^{d}}\right)^{1 / d^{2}} \ldots
$$

Thus,

$$
\frac{1}{\zeta}=t \cdot\left(\frac{1}{t^{d} P(1 / t)-a w t^{d-1}}\right)^{1 / d} \cdot\left(\frac{1}{t_{1}^{d} P\left(1 / t_{1}\right)-a w_{1} t_{1}^{d-1}}\right)^{1 / d^{2}} \ldots
$$

where the $d^{n}$ th roots symbols mean the standard branch of the root. To simplify, we will use the notation $1 / \zeta+t \cdot P_{1}(t, w) . P_{2}(t, w) \ldots$ Alternatively, one can write 
$1 / \zeta=t . \exp \left(\frac{\ln \left(\frac{1}{t^{d} P(1 / t)-a w t}\right)}{d}+\frac{\ln \left(\frac{1}{t_{1}^{d} P\left(1 / t_{1}\right)-a w_{1} t_{1}^{d-1}}\right)}{d^{2}}+\ldots\right)$, and observe that the convergence comes from the fact that all the terms $\ln \left(\frac{1}{t_{n}^{d} P\left(1 / t_{n}\right)-a w_{n} t_{n}^{d-1}}\right)$ are bounded. We can then expand each term and rewrite the whole product $P_{1} \cdot P_{2} \ldots$ as a single power series $P(t, w)$. We claim now that $P(0, w)$ is identically equal to 1 . Indeed, it is easy to check by induction that $t_{n}=0 \Rightarrow t_{n+1}=0$ and thus $t=0$ implies that each term $P_{n}(t, w)=1$ for $n \geq 1$. Thus, one can rewrite $P(t, w)$ in the form $1+t \alpha(t, w)$ which is what we wanted.

Lemma 4.6 The function $x(\zeta, y)$ is given by

$$
x(\zeta, y)=\zeta\left(1+\frac{C}{\zeta}+U_{2}\left(\frac{1}{\zeta}, \frac{y}{\zeta}\right)\right.
$$

where $U_{2}(r, s)$ is a power series in $r, s$ which is a sum of monomials of degree at least 2.

Proof We already know that $(t, w) \mapsto(r, s)$ is a local biholomorphism tangent to the identity; therefore, one can write $t=r+T_{2}(r, s)$ where $T_{2}$ is a power series with monomials of total degree $D \geq 2$ and similarly $w=s+S_{2}(r, s)$. Thus, $\frac{1}{\zeta}=t(1+t \alpha(t, w))$ can be rewritten as $\frac{x}{\zeta}=1+t \alpha(t, w)=1+\left(r+T_{2}(r, s)\right) \beta(r, s)$, where $\beta(r, s)$ is the power series obtained by substituting $t=r+T_{2}(r, s)$ and $w=s+S_{2}(r, s)$ into $\alpha(t, w)$. The constant $\mathrm{C}$ is simply the constant term (if any) of $\beta$.

Lemma 4.7 (Laurent series for $x(\zeta, y))$ One has

$$
x(\zeta, y)=\left(\zeta+\frac{C}{\zeta}+\cdots\right)+y\left(\frac{A_{1}}{\zeta^{m_{1}}}+\cdots\right)+\cdots+y^{n}\left(\frac{A_{n}}{\zeta^{m_{n}}}+\cdots\right)+\cdots
$$

where $m_{1}=1$ and $m_{n}=n-1$ for $n \geq 2$.

Proof This is an immediate consequence of the previous lemma.

We recall that the Hénon map can be written in the coordinates $(\zeta, z)$ as $H:(\zeta, z) \mapsto\left(\zeta^{d}, \frac{a}{d} z+Q(\zeta)\right)$.

Lemma 4.8 In the Laurent series expansion $x(\zeta, y)=h_{0}(\zeta)+y h_{1}(\zeta)+y^{2} h_{2}(\zeta)+\cdots$, one needs only $h_{0}$ to determine $Q(\zeta)$.

Proof We recall that $Q(\zeta)$ is given by the $($ degree $d+1)$ polynomial part of

$$
S(\zeta)=\zeta^{d} \int_{0}^{x(\zeta, 0)} \frac{\partial x}{\partial \zeta}\left(\zeta^{d}, u\right) \mathrm{d} u
$$


Now

$$
\frac{\partial x}{\partial \zeta}=\left(1-\frac{C}{\zeta^{2}}+\cdots\right)+y\left(\frac{B_{1}}{\zeta^{m_{1}+1}}+\cdots\right)+\cdots+y^{n}\left(\frac{B_{n}}{\zeta^{m_{n}+1}}+\cdots\right)+\cdots
$$

and

$$
\begin{aligned}
\int_{0}^{y} \frac{\partial x}{\partial \zeta}\left(\zeta^{d}, u\right) \mathrm{d} u= & y\left(1-\frac{C}{\zeta^{2 d}}+\cdots\right)+y^{2}\left(\frac{C_{1}}{\zeta^{d\left(m_{1}+1\right)}}+\cdots\right) \\
& +\cdots+y^{n+1}\left(\frac{C_{n}}{\zeta^{d\left(m_{n}+1\right)}}+\cdots\right)+\cdots
\end{aligned}
$$

Finally, one substitutes $x(\zeta, 0)=\zeta+\frac{C}{\zeta}+\cdots$ in $y$ and multiply by $\zeta^{d}$. Thus, we can get rid in $\int_{0}^{x(\zeta, 0)} \frac{\partial x}{\partial \zeta}\left(\zeta^{d}, u\right) \mathrm{d} u$ of all the terms $\frac{1}{\zeta^{k}}, k \geq d$. Now for $n=1$, we have a leading term $\zeta^{2} \cdot \frac{C_{1}}{\zeta^{2 d}}$, but since for any $d \geq 2$ we have that $2 d-2 \geq d$ we can get rid of this term (and all the following terms in $\left.h_{1}(\zeta)\right)$. Also for any $n \geq 2$ we have a leading term $\zeta^{n+1} \cdot \frac{C_{n}}{\zeta^{d .\left(m_{n}+1\right)}}$. Now $d \cdot\left(m_{n}+1\right)-(n+1)=d \cdot n-(n+1)=d+d \cdot(n-1)-(n+1) \geq$ $d+2 \cdot(n-1)-(n+1) \geq d+n-3 \geq d$.

Thus we can neglect all the terms coming from $h_{n}(\zeta)$.

Determining how many terms are needed in the expansion.

Low-order examples.

Lemma 4.9 The function $Q(\zeta)$ is given by the polynomial part of the product $\zeta^{d} \cdot x(\zeta, 0)$.

Proof We already saw that $\int_{0}^{y} \frac{\partial x}{\partial \zeta}\left(\zeta^{d}, u\right) \mathrm{d} u$ can be written as

$$
y\left(1-\frac{C}{\zeta^{2 d}}+\cdots\right)+y^{2}\left(\frac{C_{1}}{\zeta^{d\left(m_{1}+1\right)}}+\cdots\right)+\cdots+y^{n+1}\left(\frac{C_{n}}{\zeta^{d\left(m_{n}+1\right)}}+\cdots\right)+\cdots
$$

and that the terms corresponding to $y^{k}, k \geq 2$ can be dropped. Thus, $Q(\zeta)$ is given by the polynomial part of $\zeta^{d} \cdot x(\zeta, 0) \cdot\left(1-\frac{C}{\zeta^{2 d}}+\cdots\right)$, which is the same as the polynomial part of $\zeta^{d} \cdot x(\zeta, 0)$ because $x(\zeta, 0)$ is of order $\zeta$ and therefore we can drop the term $\frac{C}{\zeta^{2 d}}$ and the higher order ones in $\frac{1}{\zeta}$.

We now relate $x(\zeta, 0)$ to $\zeta(x, 0)$ :

Lemma 4.10 Assume that

$$
\zeta(x, 0)=x+\frac{D_{1}}{x}+\frac{D_{2}}{x^{2}}+\frac{D_{3}}{x^{3}}+\frac{D_{4}}{x^{4}}+\frac{D_{5}}{x^{5}}+\cdots
$$

Then

$$
x(\zeta, 0)=\zeta+\frac{F_{1}}{\zeta}+\frac{F_{2}}{\zeta^{2}}+\frac{F_{3}}{\zeta^{3}}+\frac{F_{4}}{\zeta^{4}}+\frac{F_{5}}{\zeta^{5}}+\cdots,
$$

where the coefficients $F_{i}$ are given by 
1. $F_{1}=-D_{1}$,

2. $F_{2}=-D_{2}$,

3. $F_{3}=-D_{3}-D_{1}^{2}$,

4. $F_{4}=-3 D_{1} D_{2}-D_{4}$,

5. $F_{5}=-2 D_{1}^{3}-2 D_{2}^{2}-4 D_{1} D_{3}-D_{5}$.

Proof First we have

$$
\frac{1}{\zeta}=\frac{1}{x} \cdot\left(1-\frac{D_{1}}{x^{2}}-\frac{D_{2}}{x^{3}}+\frac{-D_{3}+D_{1}^{2}}{x^{4}}\right)+O\left(\frac{1}{x^{6}}\right) .
$$

Similarly,

$$
\begin{aligned}
& \frac{1}{\zeta^{2}}=\frac{1}{x^{2}} \cdot\left(1-\frac{2 D_{1}}{x^{2}}-\frac{2 D_{2}}{x^{3}}\right)+O\left(\frac{1}{x^{6}}\right), \\
& \frac{1}{\zeta^{3}}=\frac{1}{x^{3}} \cdot\left(1-\frac{3 D_{1}}{x^{2}}\right)+O\left(\frac{1}{x^{6}}\right), \\
& \frac{1}{\zeta^{4}}=\frac{1}{x^{4}}+O\left(\frac{1}{x^{6}}\right), \\
& \frac{1}{\zeta^{5}}=\frac{1}{x^{5}}+O\left(\frac{1}{x^{6}}\right) .
\end{aligned}
$$

Thus, after substituting these expressions in the expression of $x(\zeta, 0)$ and identifying with $x$ we obtain the following relations between the coefficients:

1. $D_{1}+F_{1}=0$,

2. $D_{2}+F_{2}=0$,

3. $D_{3}-F_{1} D_{1}+F_{3}=0$,

4. $D_{4}-F_{1} D_{2}-F_{2}\left(2 D_{1}\right)+F_{4}=0$,

5. $D_{5}+F_{1}\left(-D_{3}+D_{1}^{2}\right)-2 D_{2} F_{2}-3 D_{1} F_{3}+F_{5}=0$.

From those, we deduce

1. $F_{1}=-D_{1}$,

2. $F_{2}=-D_{2}$,

3. $F_{3}=-D_{3}-D_{1}^{2}$,

4. $F_{4}=-3 D_{1} D_{2}-D_{4}$,

5. $F_{5}=-2 D_{1}^{3}-2 D_{2}^{2}-4 D_{1} D_{3}-D_{5}$.

It remains to find the expansion for $x(\zeta, 0)$.

Lemma 4.11 We assume that the Hénon map is given by $H(x, y)=(P(x)-a y, x)$ with $P$ being a degree $d$ polynomial that is centered (i.e., without degree $d-1$ term). Consider $H^{n}(x, y)=\left(x_{n}(x, y), x_{n-1}(x, y)\right)$. Then the function $x_{n}$ satisfies the following: 
1. $x_{n}=x_{n}(x, 0)$ is a degree $d^{n}$ polynomial of the form $x_{n}(x, 0)=x^{d^{n}}+\alpha_{n} x^{d^{n}-2}+\ldots$

2. $\left(\frac{P\left(x_{n}\right)-a \cdot x_{n-1}}{x_{n}^{d}}\right)^{\frac{1}{d^{n+1}}}=1+O\left(\frac{1}{x^{d^{n}-2}}\right)$.

Proof The first claim is proved by induction. For the second, we need several observations. First we have $P\left(x_{n}\right) / x_{n}^{d}=1+\frac{\alpha_{n-2}}{x_{n}^{2}}+\cdots+\frac{\alpha_{0}}{x_{n}^{d}}$. Then one observes that $\frac{1}{x_{n}^{k}}=O\left(\frac{1}{x^{k \cdot d^{n}}}\right)$, and thus $P\left(x_{n}\right) / x_{n}^{d}$ can be written as $1+O\left(\frac{1}{x^{2 \cdot d^{n}}}\right)$. For the term $\frac{a x_{n-1}}{x_{n}^{d}}$, we simply observe that it can be written as $1+O\left(1 / x^{d^{n+1}-d^{n-1}}\right)$. Finally, one can see that $d^{n+1}-d^{n-1}=d^{n-1}(d+1)(d-1) \geq d^{n-1}(d)(d-1)=d^{n}(d-1) \geq d^{n}$ when $d \geq 2$. Thus, $\left(\frac{P\left(x_{n}\right)-a \cdot x_{n-1}}{x_{n}^{d}}\right)=1+O\left(\frac{1}{x^{d^{n}-2}}\right)$ and $\left(\frac{P\left(x_{n}\right)-a \cdot x_{n-1}}{x_{n}^{d}}\right)^{\frac{1}{d^{n+1}}}=1+O\left(\frac{1}{x^{d^{n}-2}}\right)$.

Lemma 4.12 In order to determine $Q(\zeta)$, one only needs the first two terms $x\left(\frac{P(x)}{x^{d}}\right)^{1 / d}$ in the infinite product expansion of $\zeta(x, 0)$.

Proof Due to the particular form of $Q(\zeta)$, we need to use in $x(\zeta, 0)$ only the terms up to order $1 / \zeta^{d-1}$. Thus, in $\zeta(x, 0)$ we only need to keep the terms up to the order $1 / \zeta^{d-1}$. Now for any $n \geq 2$ we have $d^{n}-2 \geq d^{2}-2>d-1$ (as soon as $d \geq 2$ ).

Lemma 4.13 (Low-order examples)

(1) Degree 2: Given that $P(x)=x^{2}+$ cone has $\zeta(x, 0)=x+\frac{c}{2 x}+O\left(1 / x^{2}\right)$.

(2) Degree 3: For $P(x)=x^{3}+A x+B$ one has $\zeta(x, 0)=x+\frac{A}{3 x}+\frac{B}{3 x^{2}}+O\left(1 / x^{3}\right)$.

(3) Degree 4: For $P(x)=x^{4}+A x^{2}+B x+C$, one has

$$
\zeta(x, 0)=x+\frac{A}{4 x}+\frac{B}{4 x^{2}}+\frac{8 C-3 A^{2}}{32 x^{3}}+O\left(1 / x^{4}\right) .
$$

(4) Degree 5: For $P(x)=x^{5}+A x^{3}+B x^{2}+C x+D$, one has

$$
\zeta(x, 0)=x+\frac{A}{5 x}+\frac{B}{5 x^{2}}+\frac{5 C-2 A^{2}}{25 x^{3}}+\frac{D}{5 x^{4}}+O\left(1 / x^{5}\right) .
$$

(5) Degree 6: For $P(x)=x^{6}+A x^{4}+B x^{3}+C x^{2}+D x+E$, one has

$$
\begin{aligned}
\zeta(x, 0) & =x+\frac{A}{6 x}+\frac{B}{6 x^{2}}+\frac{12 C-5 A^{2}}{72 x^{3}}+\frac{12 D-10 A B}{72 x^{4}} \\
+ & \frac{1}{x^{5}} \cdot\left(\frac{E}{6}-\frac{5 B^{2}}{72}-\frac{10 A C}{72}+\frac{55 A^{3}}{1296}\right)+O\left(1 / x^{6}\right)
\end{aligned}
$$

Putting things together Now we have everything we need to express the Hénon map in the new coordinates. The final theorem is a direct consequence of the previous lemmas.

Theorem 4.14 In the coordinates $(\zeta, z)$, the Hénon map is given by

$$
H(\zeta, z)=\left(\begin{array}{c}
\zeta^{d} \\
\frac{a}{d} z+Q(\zeta)
\end{array}\right)
$$

where the degree $(d+1)$ polynomial (without constant term) $Q$ is as follows: 
(1) Degree 2: Given that $P(x)=x^{2}+c$ one has $Q(\zeta)=\zeta^{3}-\frac{c}{2} \zeta$.

(2) Degree 3: For $P(x)=x^{3}+A x+B$ one has $Q(\zeta)=\zeta^{4}-\frac{A}{3} \zeta^{2}-\frac{B}{3} \zeta$.

(3) Degree 4: For $P(x)=x^{4}+A x^{2}+B x+C$, one has

$$
Q(\zeta)=\zeta^{5}-\frac{A}{4} \zeta^{3}-\frac{B}{4} \zeta^{2}+\frac{A^{2}-8 C}{32} \zeta
$$

(4) Degree 5: For $P(x)=x^{5}+A x^{3}+B x^{2}+C x+D$, one has

$$
Q(\zeta)=\zeta^{6}-\frac{A}{5} \zeta^{4}-\frac{B}{5} \zeta^{3}+\frac{A^{2}-5 C}{25} \zeta^{2}-\frac{3 A B+5 D}{25} \zeta .
$$

(5) Degree 6: For $P(x)=x^{6}+A x^{4}+B x^{3}+C x^{2}+D x+E$, one has

$$
Q(\zeta)=\zeta^{7}-\frac{A}{6} \zeta^{5}-\frac{B}{6} \zeta^{4}+\frac{A^{2}-4 C}{24} \zeta^{3}+\frac{A B-3 D}{18} \zeta^{2}+\alpha \cdot \zeta,
$$

where the coefficient $\alpha$ is given by

$$
-\frac{2 A^{3}}{6^{3}}-\frac{B^{2}}{18}-\frac{12 A C-5 A^{3}}{108}-\frac{E}{6}+\frac{5 B^{2}}{72} \frac{10 A C}{72}-\frac{55 A^{3}}{1296} .
$$

\section{Publisher's Note}

Springer Nature remains neutral with regard to jurisdictional claims in published maps and institutional affiliations.

Received: 27 July 2017 Accepted: 20 October 2017

Published online: 10 November 2017

\section{References}

1. Bedford, E., Smillie, J.: Polynomial diffeomorphisms of C2 $\mathbf{C}^{2}$, Vl: connectivity of J. Ann. of Math. 148, 695-735 (1998)

2. Bedford, E., Smillie, J.: Polynomial diffeomorphisms of $\mathbf{C}^{2}$, VIl: hyperbolicity and external rays. Ann. Sci. École Norm. Sup. 4(32), 455-497 (1999)

3. Bousch, T.: Automorphismes des applications de Hénon (1994) (unpublished manuscript)

4. Favre, C.: Classification of 2-dimensional contracting rigid germs and Kato surfaces. I. J. Math. Pures Appl. 9(79), 475-514 (2000)

5. Fornæss, J., Sibony, N.: Complex Hénon mappings in $\mathbf{C}^{2}$ and Fatou-Bieberbach domains. Duke Math. J. 65, 345-380 (1992)

6. Hubbard, J.H., Oberste-Vorth, R.W.: Hénon mappings in the complex domain, I: the global topology of dynamical space. Inst. Hautes Etudes Sci. Publi. Math. 79, 5-46 (1994)

7. Hubbard, J.H., Oberste-Vorth, R.W.: Hénon mappings in the complex domain, II, projective and inductive limits of polynomials. Real and complex dynamical systems (Hillerød, 1993). NATO Adv. Sci. Inst. Ser. C Math. Phys. Sci. 464 89-132 (1995)

8. Hubbard, J.H., Papadopol, P., Veselov, V.: A compactification of Hénon mappings in $\mathbf{C}^{2}$ as dynamical systems. Acta Math. 184, 203-270 (2000)

9. Morosawa, S., Nishimura, Y., Taniguchi, M., Ueda, T.: Holomorphic Dynamics. Cambridge University Press, Cambridge (2000) 